\title{
Coherent whistler emissions in the magnetosphere - Cluster observations
}

\author{
E. M. Dubinin ${ }^{1}$, M. Maksimovic ${ }^{2}$, N. Cornilleau-Wehrlin ${ }^{3}$, D. Fontaine ${ }^{3}$, P. Travnicek ${ }^{4}$, A. Mangeney ${ }^{2}$, \\ O. Alexandrova ${ }^{2}$, K. Sauer ${ }^{1}$, M. Fraenz ${ }^{1}$, I. Dandouras ${ }^{5}$, E. Lucek ${ }^{6}$, A. Fazakerley ${ }^{7}$, A. Balogh ${ }^{6}$, and M. Andre ${ }^{8}$ \\ ${ }^{1}$ Max-Planck-Institute für Sonnensystemforschung, Lindau, Germany \\ ${ }^{2}$ LESIA, Observatoire de Paris, France \\ ${ }^{3}$ CETP/IPSL, Velizy, France \\ ${ }^{4}$ Institute of Atmospheric Physics, Prague, Czech Republic \\ ${ }^{5} \mathrm{CESR}$, Toulouse, France \\ ${ }^{6}$ Imperial College, London, UK \\ ${ }^{7}$ Mullard Space Sci.Lab. Surey, UK \\ ${ }^{8}$ Swedish Institute of Space Physics, Uppsalla, Sweden
}

Received: 27 July 2006 - Accepted: 22 January 2007 - Published: 1 February 2007

\begin{abstract}
The STAFF-SC observations complemented by the data from other instruments on Cluster spacecraft were used to study the main properties of magnetospheric lion roars: sporadic bursts of whistler emissions at $f \sim 0.1-0.2 f_{e}$ where $f_{e}$ is the electron gyrofrequency. Magnetospheric lion roars are shown to be similar to the emissions in the magnetosheath while the conditions for their generation are much less favorable: the growth rate of the cyclotron temperature anisotropy instability is much smaller due to a smaller number of the resonant electrons. This implies a nonlinear mechanism of generation of the observed wave emissions. It is shown that the observed whistler turbulence, in reality, consists of many nearly monochromatic wave packets. It is suggested that these structures are nonlinear Gendrin's whistler solitary waves. Properties of these waves are widely discussed. Since the group velocity of Gendrin's waves is aligned with the magnetic field, these well guided wave packets can propagate through many magnetic "bottles" associated with mirror structures, without being trapped.
\end{abstract}

Keywords. Magnetospheric physics (Plasma waves and instabilities) - Radio science (Nonlinear phenomena; Waves in plasma)

\section{Introduction}

Intense bursts of electromagnetic waves with a center frequency near $100 \mathrm{~Hz}$ (which corresponds to $0.1-0.5 \Omega_{e}$ where $\Omega_{e}$ is the electron cyclotron frequency) were first detected

Correspondence to: E. M. Dubinin

(dubinin@mps.mpg.de) in the magnetosheath by the OGO-5 satellite (Smith et al., 1969, 1971). These emissions were called "lion roars" and are a characteristic feature of the wave activity in the magnetosheath. Smith and Tsurutani (1976) identified lion roars as right-handed circularly polarized waves (whistlers) propagating at angles of less than $30^{\circ}$ to the magnetic field. The lion roars are often closely associated with troughs in the magnetic field amplitude and enhancements in the plasma density: attributed to mirror modes excited by the ion pressure anisotropy downstream of the bow shock (Tsurutani et al., 1982). Analysis of the simultaneous wave and particle observations made by ISEE-1 showed that lion roars are generated by the cyclotron resonant instability in the magnetosheath plasma under conditions of electron temperature anisotropy $T_{e \perp}>T_{e \|}$, where $T_{e \perp}$ and $T_{e \|}$ are the perpendicular and parallel to the magnetic field temperatures (Thorne and Tsurutani, 1981). Over the observed whistler frequency range the resonant electrons have energies $E \geq E_{r}\left(1-\frac{\omega}{\Omega_{e}}\right)^{3} \frac{\Omega_{e}}{\omega}$. Here $E_{r}$ is the characteristic magnetic field energy per electron, $E_{r}=\frac{B^{2}}{2 \mu_{o} n_{e}}=\frac{m_{e} V_{A e}^{2}}{2}$, and $\omega$ and $\Omega_{e}$ are the characteristic frequency of the emissions and electron gyrofrequency, respectively, and $V_{A e}$ is the Alfvén speed based on the electron mass density (Kennel and Thorne, 1967; Thorne and Tsurutani, 1981). This condition implies that in the magnetic field troughs, where the plasma density increases, the characteristic energy $E_{r}$ may drop down to values comparable to the thermal electron energy, and so one may expect an exponential increase in the number density of resonant electrons and thus a growth of whistler waves.

Published by Copernicus GmbH on behalf of the European Geosciences Union. 
There have been few studies of Lion roars inside the Earth's magnetosphere. Baumjohann et al. (2000) reported the presence of emissions in the magnetosphere, close to the magnetopause, that had characteristics almost identical to those of magnetosheath lion roars. In this paper we will focus on the observations of narrow-band emissions made by Cluster in the duskside magnetosphere. We will also discuss a possible origin of the coherent structure of lion roars. The actual waveform of the lion roars was unknown till the end of the 1990s when high time and frequency resolution measurements made on the Geotail and Equator-S spacecraft showed that these emissions consist of near-monochromatic packets (Zhang et al., 1988; Baumjohann et al., 1999). These two features, a coherency and a wave packet structure, are difficult to explain in terms of linear theories of cyclotron resonant instability fed by a temperature anisotropy, since the theory predicts a broader frequency range of excited waves with random phases. We suggest that the coherent structure of the wave emissions is associated with the nonlinear properties of the so-called Gendrin' whistler waves (Gendrin, 1961). A nonlinear system consisting of the electrons and protons participating in the wave motion "resonates" at a frequency at which the phase speed of the waves equals their group speed. Periodic momentum exchange between the electrons and protons, mediated by Maxwell stresses, gives rise to the wave packet structures which are a kind of solitary Gendrin wave packets.

The layout of the paper is as follows. Section 2 contains the description of the instrumentation. Section 3 contains discussion of the macroscopic plasma parameters in the regions occupied by the whistler emissions. It is found that, similar to magnetosheath lion roars, the magnetospheric narrow-banded emissions appear as intermittent bursts of waves closely associated with the magnetic field depressions. These field troughs are probably a manifestation of the mirror wave structures excited by the proton temperature anisotropy that is confirmed by the measurements. The behavior of the electrons is more complicated. A close correlation between the wave intensity and the temperature anisotropy of the bulk electron distribution is not found. On the other hand, the observed trapped population of more energetic electrons is sufficient to provide the energy source for the generation of the whistlers. The structure of the waveforms is also discussed in Sect. 3. It is shown that the "whistler turbulence" consists of coherent wave structures in the form of a train of periodic, almost monochromatic wave packets. The amplitude of the waves is almost the same as those found in the magnetosheath. A mechanism to explain the coherence of the waves and the wave packet structure is discussed in Sect. 4 .

\section{Instrumentation}

We present observations of lion roars in the magnetosphere made by the STAFF experiment on the Cluster spacecraft.
STAFF (Spatio-Temporal Analysis of Field Fluctuations) comprises of a triaxial search coil magnetometer, a waveform receiver (SC) with an upper frequency of either $10 \mathrm{~Hz}$ or $180 \mathrm{~Hz}$, and a digital spectrum analyzer (SA) with a frequency range up to $4 \mathrm{kHz}$ (Cornilleau-Wehrlin et al., 1997, 2003; Maksimovic et al., 2001). In this study we use the data acquired in the burst mode of STAFF operation when the wave form of whistler emissions can be recovered up to a frequency of $180 \mathrm{~Hz}$. The electric field component of the wave emissions is measured by the EFW experiment (Gustafsson et al., 1997). Since the presence of lion roars is favored by the magnetic field troughs associated with mirror modes, the magnetic field from FGM instrument (Balogh et al., 2001), and the ion data from the CIS experiment (Rème et al., 2001) are also used to complement the wave observations. In our study the Hot Ion Analyzer (HIA) data are used to obtain the main plasma bulk parameters. Since lion roars are probably generated by the resonant cyclotron instability associated with the electron temperature anisotropy, electron characteristics obtained from the PEACE experiment (Johnstone et al., 1997) are also analyzed. The PEACE instrument comprises Low and High-Energy Electron Analyzers (LEEA and HEEA) which measure electrons in the energy range $0.7 \mathrm{eV}-$ $26 \mathrm{keV}$ sampling the full $4 \pi$ per $4 \mathrm{~s}$ spin. Both sensors have different modes permitting different combinations of energy and angular resolution. In the standard, medium resolution mode the number of the energy sweeps is 32 sweeps/spin with 60 energy steps and an azimuthal resolution of $11.25^{\circ}$. In the burst mode the instrument performs 64 sweeps with 30 energy steps and $5.6^{\circ}$ azimuthal resolution.

\section{Observations}

\subsection{Macroscopic characteristics}

On 11 December 2001 (day 345) at 11:0014:30 UT Cluster was in the northern dusk magnetosphere at geocentric distances $\sim 14-16 R_{E}$ $\left(x_{\mathrm{GSM}} \sim(-1.5 \div 0) R_{E}, y_{\mathrm{GSM}} \sim(14 \div 16.5) R_{E}\right.$,

$\left.z_{\mathrm{GSM}} \sim(4 \div 4.5) R_{E}\right)$. The tetrahedron separation scale was of the order of $\leq 2500 \mathrm{~km}$. All four spacecraft observed sporadic bursts of rather narrow-banded wave emissions at frequencies of about $20-50 \mathrm{~Hz}$. Figure 1 shows the spectrograms of the power spectral density of the $B_{z}$ component of the magnetic field fluctuations measured by the STAFF-SC experiment on all four spacecraft. Intermittent emissions are almost always associated with magnetic troughs, clearly seen in a plot of the magnetic field strength (the white curves). This close association suggests that these emissions are examples of lion roars which are usually observed in the magnetosheath. Well correlated magnetic field signatures indicate that the four spacecraft cross almost the same magnetic field tubes, some of which are acting as wave guides. 


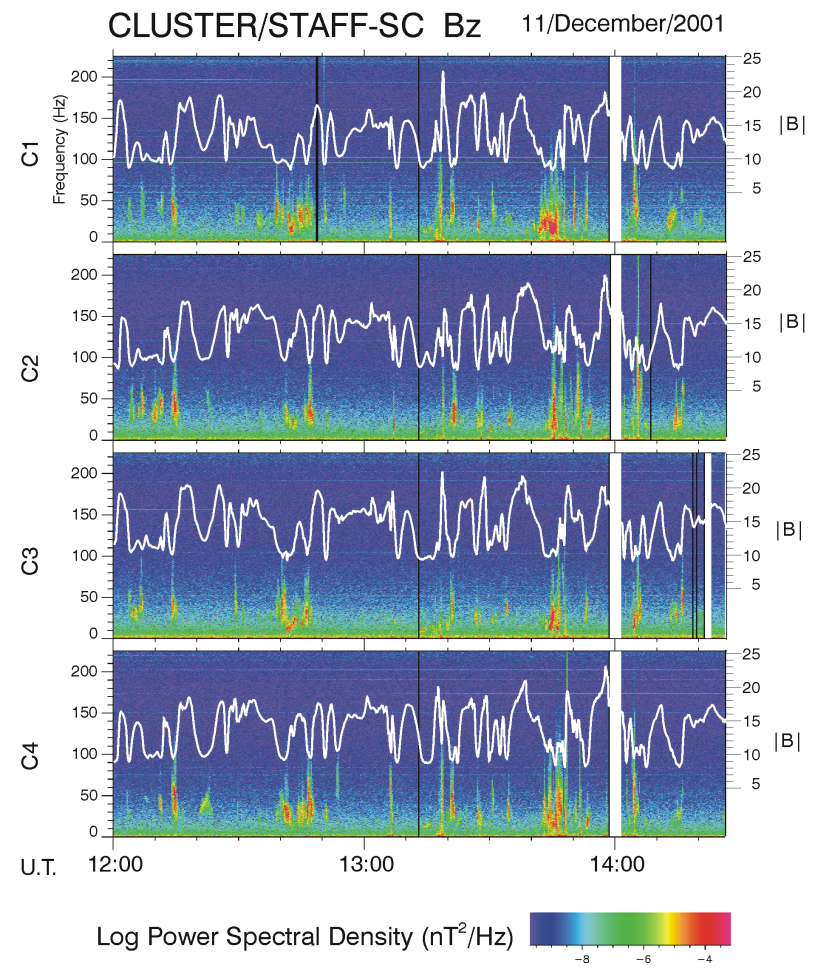

Fig. 1. Spectrograms of whistler emissions in the distant magnetosphere. The white curves depict the magnetic field strength.

\subsection{Multipoint measurements}

In the past observations of lion roars were mainly made from a single satellite. Cluster allows us to carry out simultaneous four-point measurements. Figure 2 shows a close-up of the waves and the magnetic field measured by Cluster during the time interval 13:30-13:40 UT. Spacecraft C3 records a burst of waves in the magnetic trough at $\sim 13: 34$ UT. Spacecraft $\mathrm{C} 4$ and $\mathrm{C} 2$ enter this magnetic field depleted tube later and observe the waves with a time lag $\sim 30$ s. Spacecraft $\mathrm{C} 1$ also crosses this tube at the same time as satellite $\mathrm{C} 3$ but the intensity of the waves recorded by spacecraft $\mathrm{C} 1$ is significantly smaller. Similar features are also observed in other events. For example, at 13:05-13:10 UT Cluster C1, C4, C2 successively, with a time lag of $\sim 30 \mathrm{~s}$, cross the field tubes characterized by a depression of the magnetic field and bursts of whistler emissions. The fact that spacecraft C1, C4, C2 cross the same magnetic field tubes with a time lag yet continue to observe the wave activity gives a hint that the field tube may be "wave active" for, at least, $30 \mathrm{~s}-1 \mathrm{~min}$. At the same time however, spacecraft C3 is situated in this field depleted tube, but does not observe whistler waves. Similarly, at $\sim 12: 52$ spacecraft $\mathrm{C} 3$ does not observe the wave activity although the other spacecraft do. Note that if a characteristic scale of magnetic bottles along the magnetic field is about $10^{4} \mathrm{~km}$ (see e.g. Maksimovic et al., 2001) then the transit time of whistler waves at $\omega \sim 0.1 \Omega_{e}$ is about $5 \mathrm{~s}$, and dur-

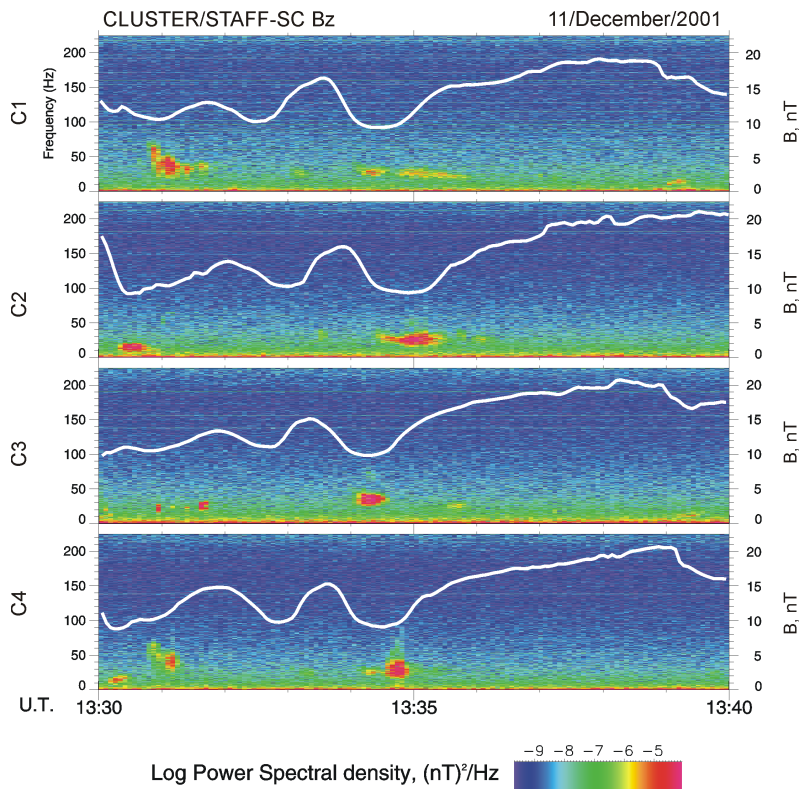

Fig. 2. Close-up of the wave intensity on different spacecraft crossing the same magnetic field depleted tubes.

ing $0.5-1$ min of a wave activity, the wave packets can either propagate across several successive magnetic bottles or be trapped in a magnetic field trough.

\subsection{Ion dynamics}

Lion roar whistlers are often associated with the presence of mirror waves characterized by anticorrelated perturbations in the density and the magnetic field magnitude. Figure 3 (the top panel) shows the proton number density and the magnetic field value measured on spacecraft $\mathrm{C} 1$ (the black and red curves, respectively). The measurements depict distinct out-of phase field-density variations. Mirror modes are readily excited in a plasma with the ion pressure anisotropy $\left(p_{i \perp}>p_{i \|}\right)$. The bottom panel in Fig. 3 presents the proton temperatures parallel and perpendicular to the magnetic field, deduced from the moments of the proton distribution functions (the green and blue curves, respectively). A clear anisotropy with $T_{p \perp} / T_{p \|}>1$ in the magnetic troughs is observed. The characteristic value of the proton beta $\beta_{p}$ is $\sim 15$.

\subsection{Electron dynamics}

It is widely believed that whistler emissions are driven by the temperature anisotropy of the resonant electrons: electrons with the energy $E \geq E_{r}\left(1-\frac{\omega}{\Omega_{e}}\right)^{3}\left(\frac{\Omega_{e}}{\omega}\right)$ (Thorne and Tsurutani, 1981). With parameters, $n \sim 0.6 \mathrm{~cm}^{-3}, B \sim 10 \mathrm{nT}$, $\omega / \Omega_{e} \sim 0.1-0.2$, the energy of the resonant electrons is about $1-2.5 \mathrm{keV}$. The PEACE data show that the bulk of the electrons have much smaller energies $\left(T_{e} \leq 200 \mathrm{eV}\right)$. It is in a contrast to a situation in the magnetosheath where 


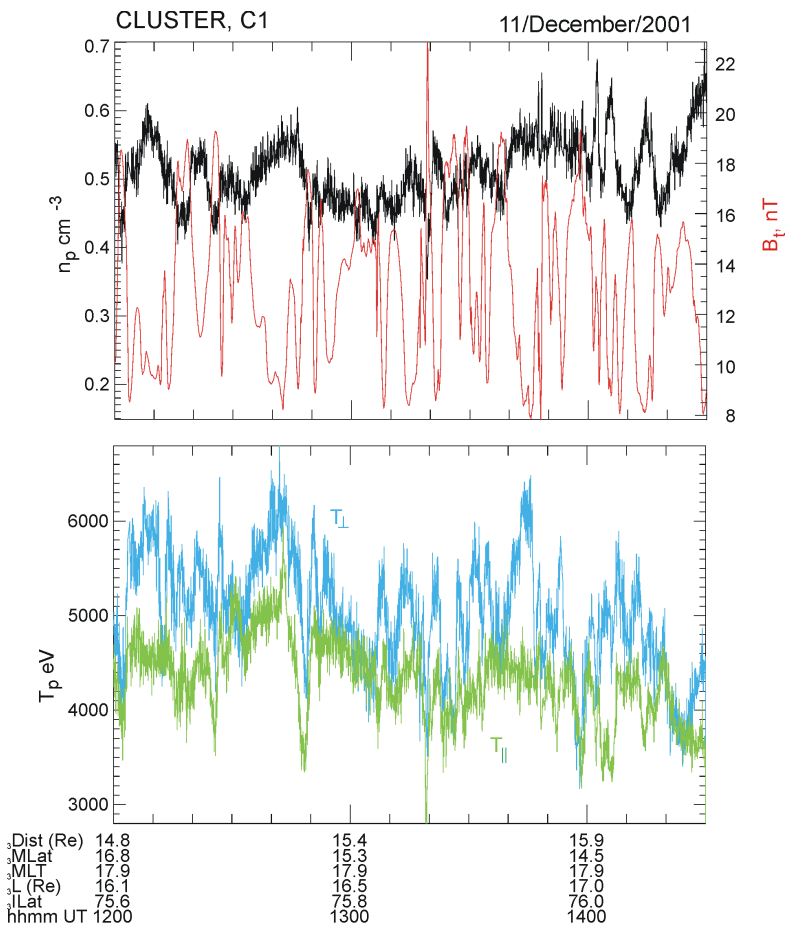

Fig. 3. (a) Density-field variations recorded by Cluster 1. (b) Proton temperatures $T_{p \|}$ and $T_{p \perp}$.

the characteristic energy of the resonant electrons is about $100 \mathrm{eV}$, i.e. close to the electron temperature, and therefore the number of resonant electrons is much higher.

Figure 4 compares the intensity of the whistler waves $(b \sim)$, the magnetic field strength and the temperature anisotropy $\left(A_{e}=T_{e \perp} / T_{e \|}\right)$ of the electrons at 14:12-14:17 UT for the four Cluster satellites derived by the fitting technique. There is no visible correlation between the wave activity and temperature anisotropy. Moreover, the factor of electron anisotropy $A_{e}$ for the bulk electron population is often less than 1 in the regions of wave activity within the magnetic field depleted tubes (e.g. the intensity of the waves recorded by spacecraft $\mathrm{C} 1$ and $\mathrm{C} 4$ reaches the maximum values in the region where $T_{e \perp} / T_{e \|}<1$ ). It is also seen that during some periods (e.g. as observed by $\mathrm{C} 1$ and C4), $T_{e \perp} / T_{e \|} \sim B / B_{o}$. Such a trend, i.e. a decrease of the anisotropy where the magnetic field decreases, might be expected if the magnetic moment $\left(T_{e \perp} / B\right)$ of the electrons and the electron temperature $T_{e \|}$ are conserved.

A different electron anisotropy signature within mirror modes was observed by ISSE-1. Tsurutani et al. (1982) showed that $T_{e \perp} / T_{e \|}$ was higher at the bottom of magnetic field troughs than at the peaks. Probably, two main factors control electron dynamics in mirror waves, (Lee et al., 1987): the conservation of the magnetic moment tends to increase $T_{e \perp}$ in the high magnetic field region while the mirror force reduces it there. The observed dominance of the first mech-
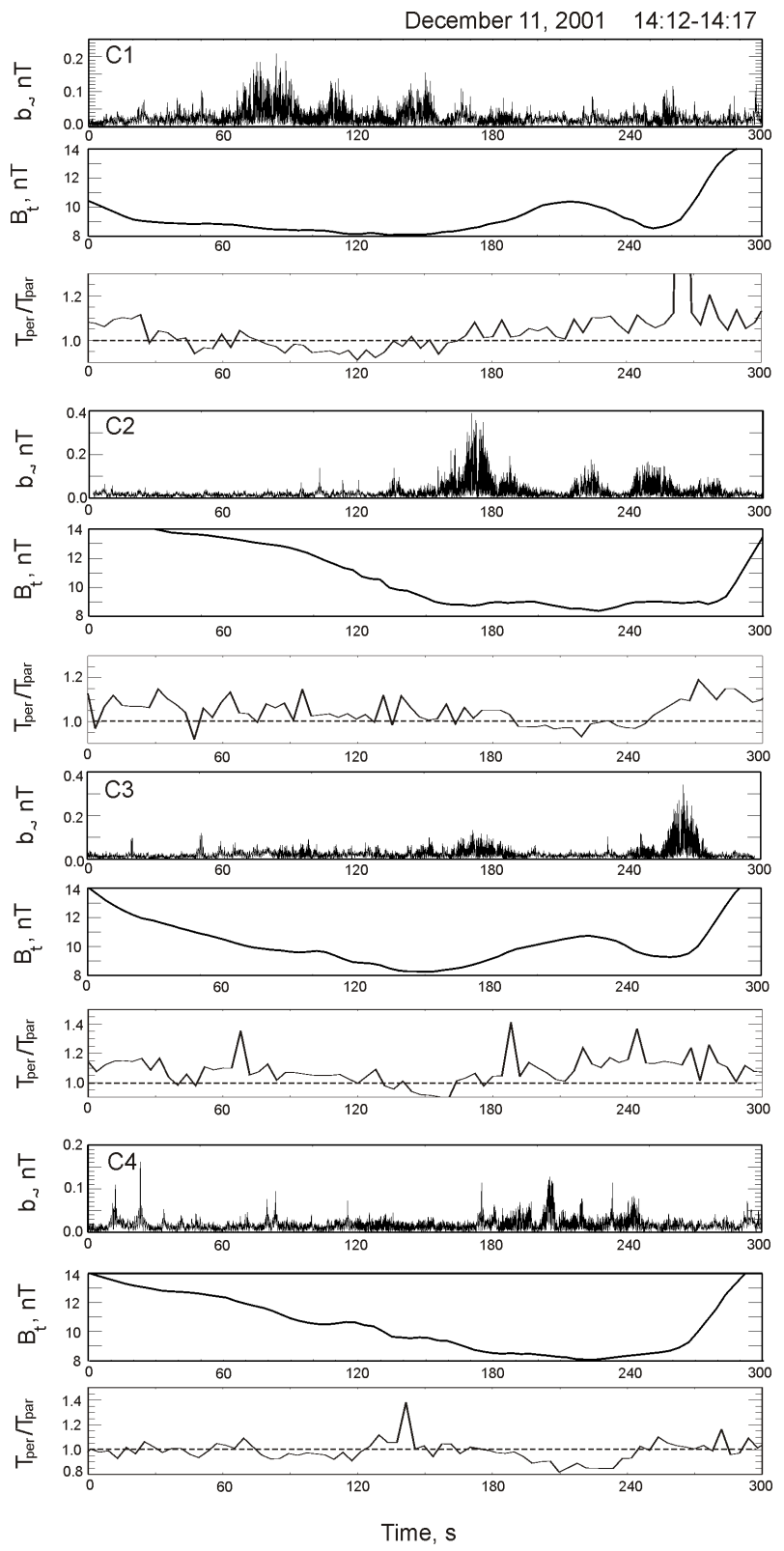

Fig. 4. Intensity of whistler emissions, the magnetic field strength and factor of the electron anisotropy $T_{e \perp} / T_{e \|}$.

anism in these Cluster observations implies an insignificant trapping in the magnetospheric troughs of the bulk of electron distribution.

However, more energetic particles, in the energy range of 1-4 keV, corresponding to the resonant electrons, clearly reveal a pitch-angle anisotropy with $T_{\perp, r} / T_{\|, r}>1$. The PEACE instrument on spacecraft $\mathrm{C} 2$ was operating in the mode which provides us with the electron fluxes in the different sectors with time resolution of $128 \mathrm{~ms}$. Figure 5 shows the wave intensity (the first panels) and the electron fluxes measured on the spacecraft $\mathrm{C} 2$ in two angular zones (the 


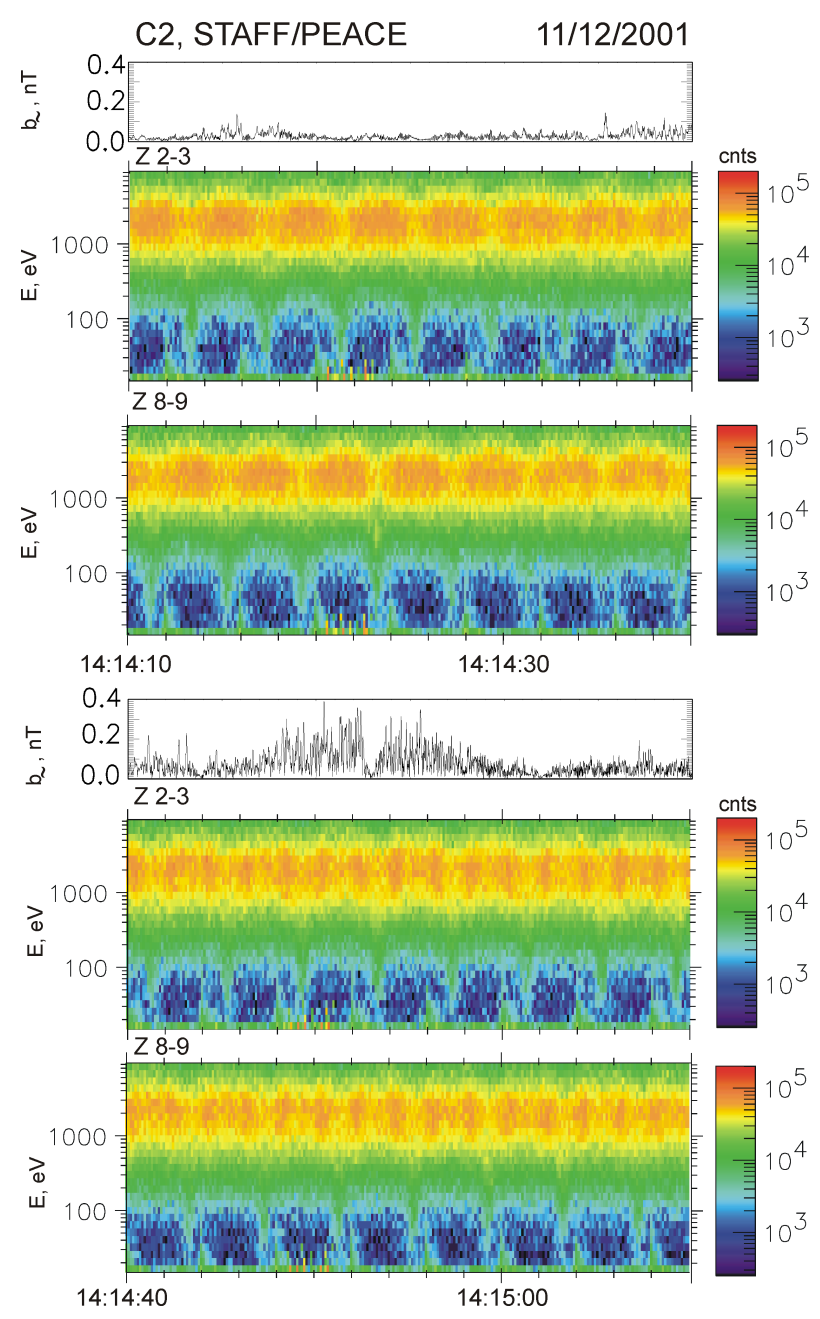

Fig. 5. Spectrogram of the electron fluxes (high resolution measurement) and the wave intensity measured onboard spacecraft 2 .

second and third panels) at 14:14:10-14:15:10 UT. Due to the spacecraft spinning (period $\sim 4$ s) zones 2-3 (8-9) have sampled respectively pitch-angles $175^{\circ}-80^{\circ}$ and $4^{\circ}-94^{\circ}$. In the magnetic field depleted tube the spacecraft clearly records signatures of the trapped electrons $\left(E_{e} \sim 1 \mathrm{keV}\right)$ and loss cones at angles close to $0^{\circ}\left(180^{\circ}\right)$ (these pitch-angles are readily identified by the presence of the field-aligned low energy electrons). The distribution of the electrons drastically changes with peaks at intermediate angles between $0^{\circ}\left(180^{\circ}\right)$ and $90^{\circ}\left(270^{\circ}\right)$ at times when the wave activity intensifies. Note that a such pitch-angle distribution differs from a distribution expected from a quasilinear diffusion in the velocity space due to a pitch-angle scattering of the electrons on whistler waves.

\subsection{Waveforms}

The remarkable feature of the wave emissions is that they consist of nearly-monochromatic wave packets. Figure 6

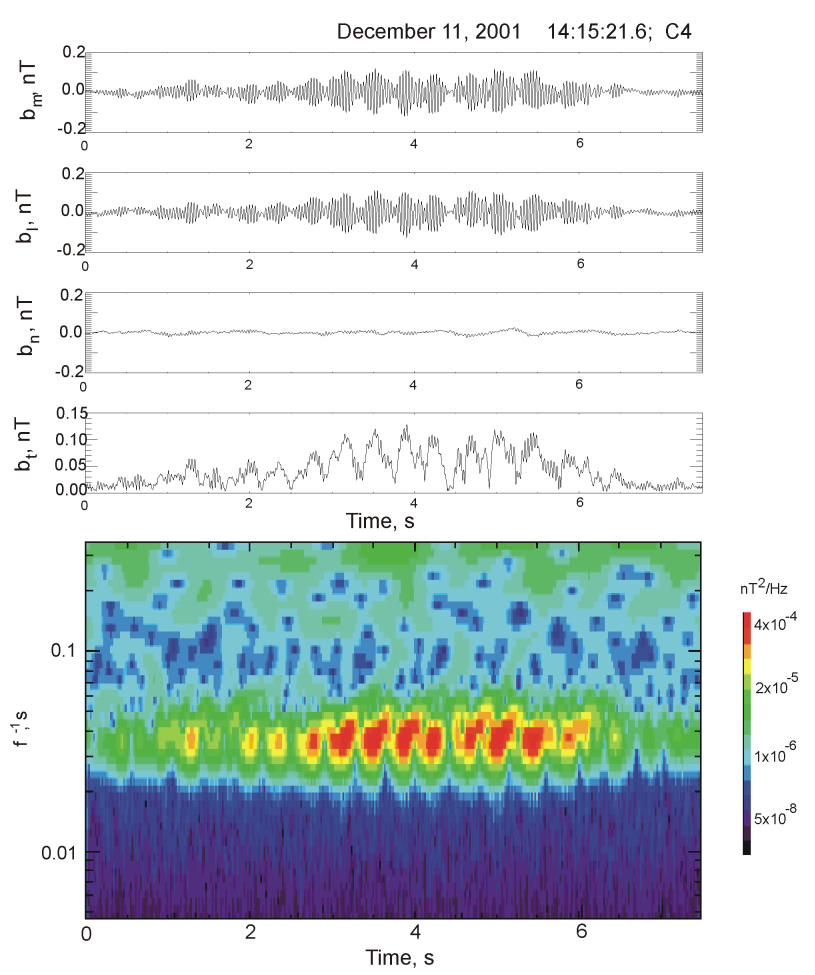

Fig. 6. The wave form of the magnetic field variations in $(m, l, n)$ coordinates. The bottom panel shows the wavelet spectrogram of the observed emissions.

shows a typical example of the magnetic field perturbations. The data are presented after applying a minimum variance analysis. It is observed that the waves are almost circularly polarized and consist of a sequence of wave packets although the variations in the magnetic field strength resemble a sequence of solitary waves. Such a periodic structure is also revealed from wavelet analysis (the bottom panel). The signal is nearly monochromatic with a center frequency near $30 \mathrm{~Hz}$ (i.e. $\sim 0.1 \Omega_{e}$ ). The number of wave cycles in the wave packets varies from $\sim 5$ to $\sim 10$ with increasing amplitude. A typical structure of a single wave packet is given in Fig. 7. The coherent character of the observed perturbations is clearly observed.

Figure 8 shows the waveforms of two mutually perpendicular components of the magnetic field and electric field perturbations measured by the STAFF-SC and EFW experiments. Wave packets observed in the magnetic and electric field variations confirm the electromagnetic origin of the emissions. The ratio $E_{\perp} / B_{\perp}$ gives us an estimate of the value of the phase speed of the waves: $v_{p h} \sim 2000-3000 \mathrm{~km} / \mathrm{s}$. Note that the Alfvén speed based on the electron mass density, $V_{A e}$, is about $10000 \mathrm{~km} / \mathrm{s}$, i.e. $v_{p h} / V_{A e} \sim 0.2-0.3$. The theory predicts phase velocities of whistler waves in this frequency range to be a factor of 2 higher. The difference could be explained by an error in the electric field values. Sometimes wave packets with several different central frequencies 

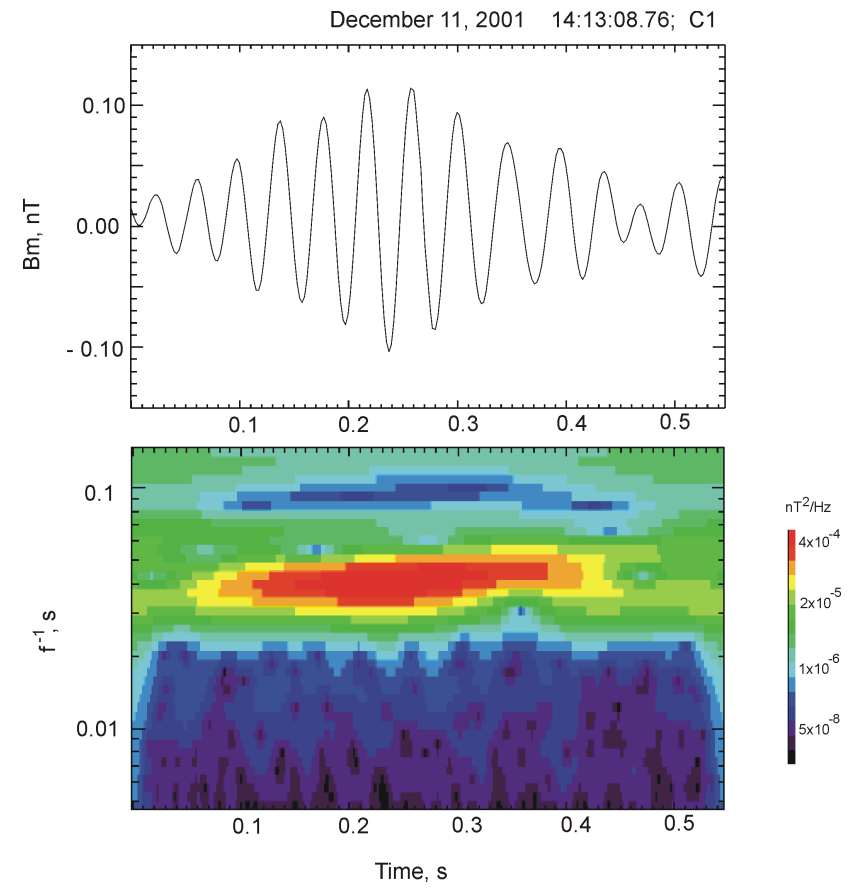

Fig. 7. The wave form of the magnetic field variations (the $B_{m}$ component) in a single wave packet. The bottom panel shows the wavelet spectrogram of the observed emissions.

are observed in the same magnetic field trough. Figure 9a depicts examples of the wave packets recorded by the spacecraft $\mathrm{C} 2$ while it remained in the same magnetic bottle for $\sim 1.5-2 \mathrm{~min}$. The characteristic frequency of the emissions lies in the range of $\sim 25-60 \mathrm{~Hz}$ although the difference in the propagation angles is rather small $\left(3.2^{\circ}\right.$ and $\left.11.5^{\circ}\right)$.

The total magnitude of the wave field often also reveals distinct oscillations. Figure $9 \mathrm{~b}$ shows the observed variations of the wave magnetic field strength in several wave packets propagating at slightly different angles $\left(15^{\circ}, 10.6^{\circ}\right.$ and $\left.8^{\circ}\right)$ to the magnetic field. It is observed that the amplitude of the small scale oscillations can be rather high.

\section{Discussion}

\subsection{Nonlinear resonance of Whistler Waves}

The nearly monochromatic and coherent character of these wave emissions, which can not be related to any plasma resonances, is difficult to explain in terms of linear instabilities. Such bounded emissions were interpreted as arising from a weak residual bump on the electron distribution function (Baumjohann et al., 1999). Baumjohann et al. (1999) assumed that only a very limited group of nearly monoenergetic electrons contributed to the growth of almost monochromatic waves. Using the linear theory of the electron cyclotron instability and the measured frequency range of the banded whistler emissions they estimated the fraction of resonant electrons to be $\sim 3 \%$ of the total electron population. It was supposed that these are just electrons trapped in mirror wave cavities. A small number of such "resonant" electrons was also found by Massood et al. (2006) who have analyzed the electron distribution functions within several events associated with the lion roars in the magnetosheath. Another interesting scenario for generation of almost monochromatic whistler waves was proposed by Treumann et al. (2000). They suggested that the mechanism of wave trapping in a mirror wave cavity selects a narrow range of wave numbers. Just one harmonic emission is expected for large $\beta_{e \|} \approx 10-20$. Note here however that in the observed magnetospheric events $\beta_{e} \leq 1$.

Although the assumption of the existence of a very small group of the "resonant" electrons (Baumjohann et al., 1999) is in a reasonable agreement with our observations of trapped electrons, we suggest another mechanism based on the nonlinear properties of whistler waves, which we call here the nonlinear resonance (NR) to distinguish from the electron cyclotron (wave/particle) resonance (ECR). Solitary structures with embedded smaller-scale oscillations which look like localized wave packets were found recently for nonlinear whistler waves (Sauer et al., 2002; Dubinin et al., 2003; Webb et al., 2005). Their existence is intimately related to a specific dispersion feature of whistlers, namely, the existence of a maximum of the phase speed $\omega / k$ at $\omega / k=V_{A e} \cos \theta / 2$, where $\theta$ is the propagation angle with respect to the magnetic field. For nonlinear whistlers of the form $f(x-U t)$ propagating parallel to the magnetic field in a cold plasma the exact analytical solutions are found in Dubinin et al. (2003); Webb et al. (2005). The important point is that in these solutions the dynamics of both species, the electrons and protons, are equally important since the amplitude of the transverse momentum flux carried by the electrons (in the wave frame) is exactly equal to the amplitude of the transverse momentum flux carried by the protons (Dubinin et al., 2003). Therefore, although the proton velocities are small $\left(v_{p} \sim v_{e} / \mu\right.$, where $\mu=m_{p} / m_{e}$ is the mass ratio) their dynamics cannot be neglected. At first glance, this result is surprising since the linear dispersion relation for whistlers at frequencies $\omega \gg \Omega_{p}$ does not contain any dependence on the proton mass $\left(\frac{k^{2}}{\omega^{2}}=\frac{1}{V_{A e}^{2}} \frac{\Omega_{e}^{2}}{\omega\left(\Omega_{e}-\omega\right)}\right)$, where $V_{A e}=B_{o} /\left(\mu_{o} n m_{e}\right)^{1 / 2}$ is the Alfvén speed based on the electron mass density. However the dynamic interplay between the electrons and protons gives rise to periodic momentum exchange between these species which leads to the appearance of an oscillatory core embedded within solitary structures.

Different solutions are found for $M_{A e}>0.5$ and $M_{A e} \leq 0.5$, where $M_{A e}=U / V_{A e}, U$ is the wave speed. Note here that $M_{A e}=0.5$ corresponds to $U=v_{p h_{\max }}=0.5 V_{A e}$, where $v_{p h_{\max }}$ is the maximum value of the phase speed of whistler waves propagating parallel to the magnetic field in a cold plasma. 


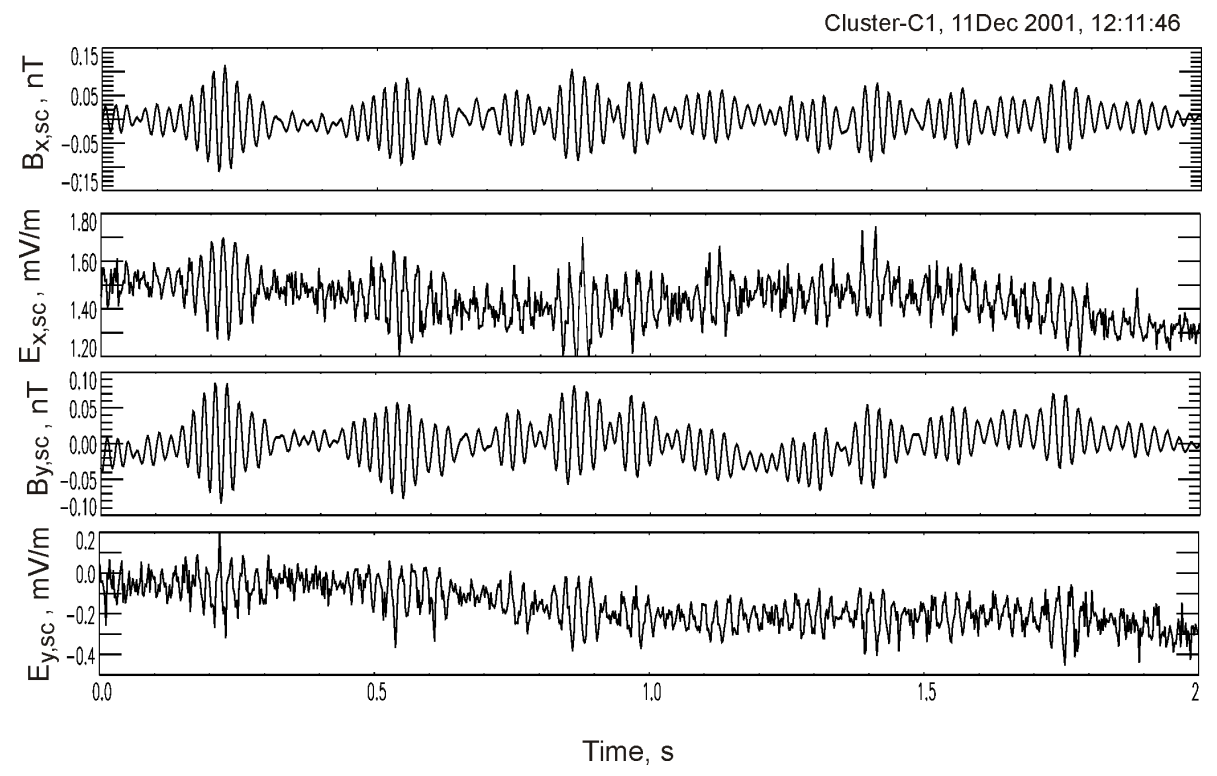

Fig. 8. The magnetic and electric field variations in the whistler wave packets.

The structure of nonlinear whistler waves propagating parallel to the magnetic field in a cold plasma can be totally described by the relation between the transverse velocity of the species (e.g. electrons, $u_{e}$ ) and the phase difference $\phi=\phi_{p}-\phi_{e}$ between the vectors of the transverse motion of the protons and electrons. In the non-relativistic case, in which charge neutrality is an appropriate assumption for the magnetosheath and distant magnetospheric plasmas, where the background magnetic field and the traveling wave velocity are parallel, the system of fully nonlinear equations can be reduced to two coupled differential equations:

$\frac{d u_{e}}{d x}=M_{A e}^{2} u_{e} \sin \phi$

$\frac{d \phi}{d x}=2 M_{A e}^{2}(1+\cos \phi)-\left(1-u_{e}^{2} / \mu\right)^{-1 / 2}$

The reader can find a more detailed analysis in Dubinin et al. (2003); Webb et al. (2005). We present here only a qualitative picture of the different types of nonlinear stationary solutions. Figure 10 shows the phase portrait of this system in the variables $u_{e}, \phi$ at $M_{A e}=0.501$ and $M_{A e}=0.5$. In the "supersonic" regime $\left(U>v_{p h_{\max }}\right)$ there is a family of periodic solutions around a circle equilibrium point limited by the trajectory connecting two saddle points at the $\phi$-axis (Fig. 10a). This latter trajectory corresponds to a soliton-type of solution. When $M_{A e} \leq 1 / 2$ the two saddle points degenerate to one at $\phi=0$ and the phase portrait reconfigures to that shown in Fig. 10b. Soliton-like solutions disappear but nonlinear stationary periodic waves remain. The remarkable feature of solitary and periodic solutions is the appearance of periodic oscillations in the transverse components of the velocities and the magnetic field embedded within solitary pulses (therefore such solitary structures were called oscillitons).

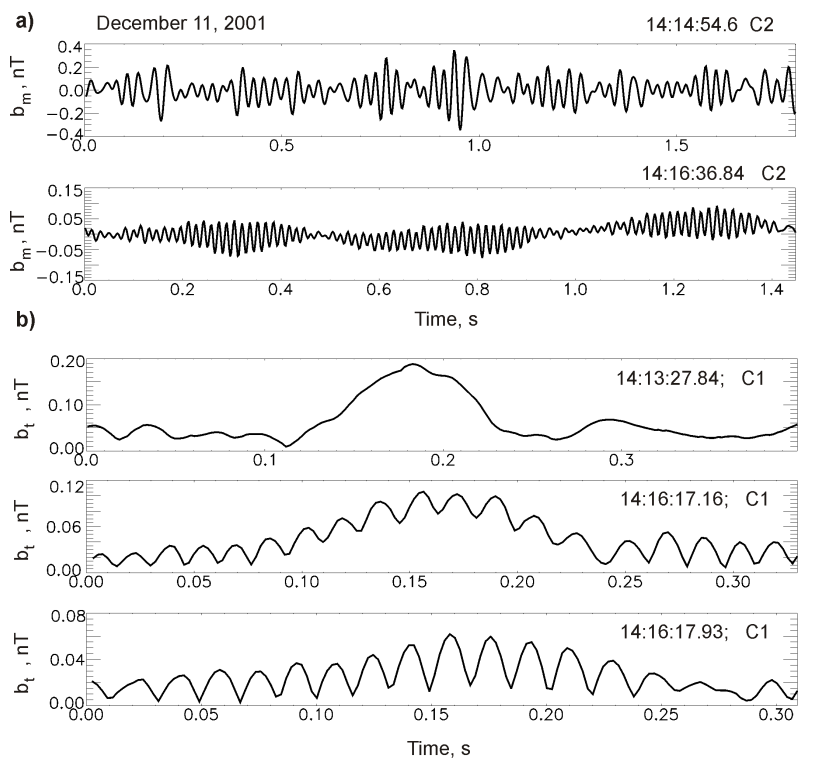

Fig. 9. (a) The magnetic field variations $\left(B_{m}\right)$ in the whistler wave packets observed in the same magnetic field trough. (b) Variations of wave amplitude in three different wave packets.

Figure 11 shows examples of a soliton (oscilliton) and a periodic sequence of wave packets corresponding to the phase portraits given in Fig. 10. The wave number of embedded oscillations is close to the wave number at which the phase speed of whistlers has a maximum, namely $k=\frac{1}{2} \frac{\Omega_{e}}{U}=\frac{\Omega_{e}}{V_{A e}}$. The appearance of small-scale oscillations can be understood from a simple analogy with two nonlinearly coupled pendulums (electron and proton oscillators) 
a)

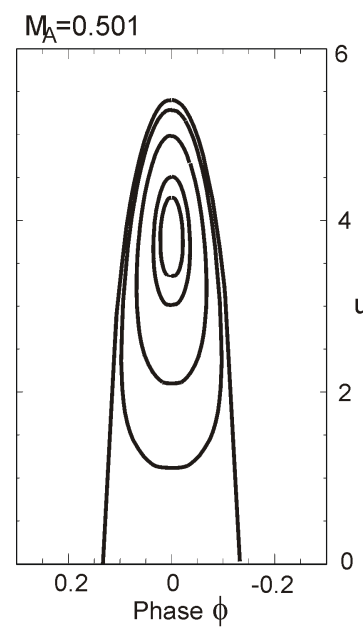

b)

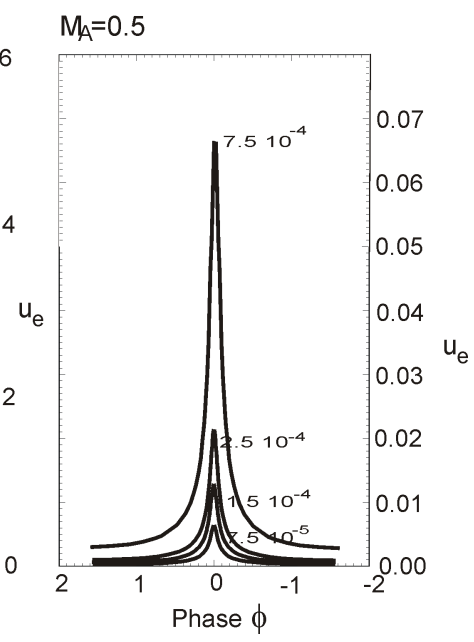

Fig. 10. (a) Phase portrait of solitary and periodic nonlinear whistler solutions in the variables $u_{e}, \phi$. (b) Phase portrait of the solutions in the form of periodic wave packets at $M_{A e}=1 / 2$.

with slightly different and varying phases. A change in the phase difference occurs due to the momentum exchange between the electrons and protons, mediated by the magnetic and electric fields. A nonlinear beating between both modes produces a localized wave packet (oscilliton) or a periodic sequence of wave packets. In the center of the oscillitons (wave packets) the "frequency" $(k U)$ of oscillations increases slightly. Such an increase can also tentatively be observed in data (see e.g. the wavelet spectrogram in Fig. 6) ${ }^{1}$ Note that the standard linear or quasilinear approximations neglect any changes in trajectories of particles in wave fields due to their momentum exchange, and therefore cannot reproduce such fully nonlinear structures.

In fact, the observed wave structures do not appear as isolated solitary waves but in trains of many wave packets. Moreover, solitary waves usually need for their excitation a large amount of a free energy (e.g. the bulk kinetic energy of the plasma must be about of $\left.n \mu U^{2} / 2 \sim n \mu V_{A e}^{2} / 8\right)$. It is easier to generate periodic wave packets than oscillitons since their speed equal to the phase speed of linear whistler waves, which can be efficiently excited by different mechanisms of whistler generation. For example, a cyclotron resonance instability driven by a temperature anisotropy or by loss-cone distribution of the electrons can excite a rather broad spectrum of emissions. The modes with the equal phase and group speeds (that occurs at $k=\Omega_{e} / V_{A e}$ and $\omega=\Omega_{e} / 2$ ) are then selected from this wave ensemble of linearly unstable

\footnotetext{
${ }^{1}$ Note that solutions in the form of wave packets also exist if only the electron dynamics is considered. Such a formal approach is sometimes used in simulations. In this case momentum exchange occurs between electrons and the wave fields.
}

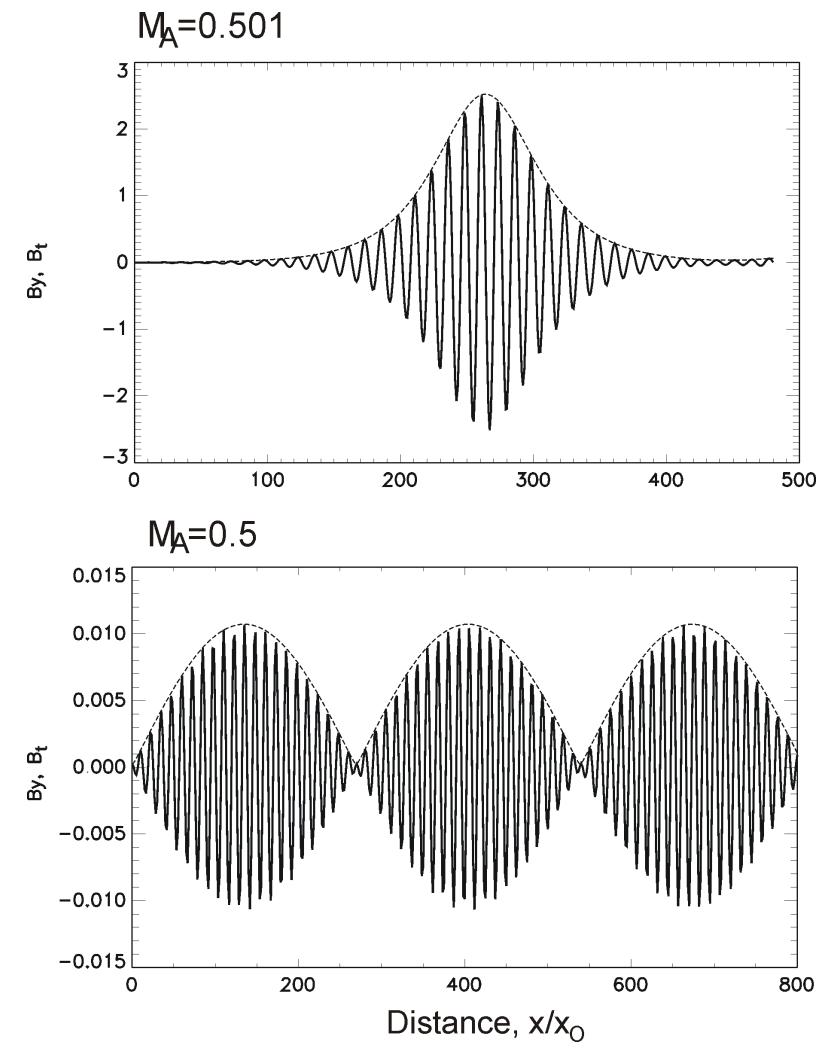

Fig. 11. The magnetic field variations (the component $B_{y}$, and the amplitude $B_{t}=\left(B_{y}^{2}+B_{z}^{2}\right)^{1 / 2}$ ) in the whistler oscilliton and periodic wave packets.

modes and amplified since the system "resonates" at this frequency. This gives rise to almost coherent and monochromatic nonlinear wave packets since the wave phase speed equals to the group speed, i.e. the speed at which energy flux supplies the structures, and the amplitude of the wave packets can reach rather large values without a significant distortion of the wave form.

Figure 12 shows the periodic wave packets generated by the system for different 'initial' conditions (different $B_{y o}$ values and $B_{z o}=0$ ). The amplitude of waves is proportional to $B_{y o}$. The amplification factor is $\sim 25$. Note that the corresponding phase portraits $\left(u_{e}, \phi\right)$ (Fig. 10b), in a certain sense, can be considered as the resonance curves. It is observed that the resonance is acute and that the selection of the "resonant" wavelength and the wave speed is rather strict. Indeed, although at $M_{A e}<1 / 2$ the phase portrait of the nonlinear system is similar to the phase portrait shown in Fig. 10b, the amplitude of the wave packets strongly decreases with deviation from $M_{A e}=1 / 2$ (Fig. 13) confirming the resonant properties of the system at $\omega=\Omega_{e} / 2$.

Such a NR can be responsible for the selection and generation of the observed coherent whistler emissions in the form of wave packets. This resonance appears in the fluid treatment and therefore differs from a classical wave-particle 


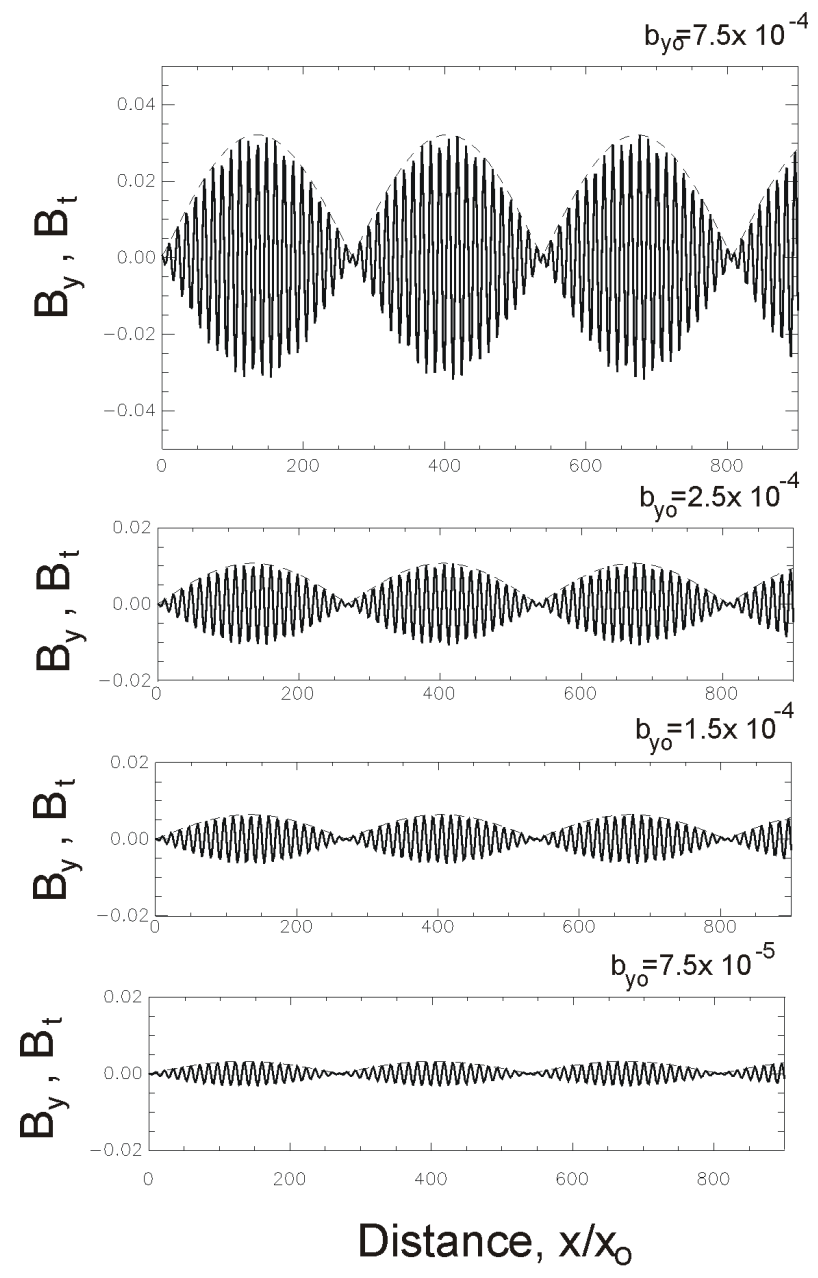

Fig. 12. Amplitude of periodic wave packets is proportional to the "initial" triggering perturbations.

resonance, for example ECR, responsible for the generation of seed whistler wave modes which can be subsequently amplified by the NR. Correspondingly, a narrow wave band of whistler waves is not an effect of a very small group of resonant (in a sense of the ECR) electrons. In a contrast, in a cold plasma the whole electron fluid oscillates at this frequency.

In the case of obliquely propagating waves the full system of nonlinear equations becomes more complicated but the main results remain similar, although now the variations of the longitudinal velocities $u_{p x}, u_{e x}$ and the magnetic field strength also contain small oscillations at $k=\Omega_{e} / V_{A e}$. It is interesting to note that the phase speed of whistlers propagating at angle $\theta$ to the magnetic field has a maximum at $\omega=\frac{\Omega_{e}}{2} \cos \theta$. It was shown (Gendrin, 1961; McKenzie, 1967) that the group velocity of such waves is directed along the magnetic field and coincides with the component of the phase speed parallel to the magnetic field. Therefore nonlinear periodic whistler wave packets for which $\cos \theta=\frac{2 \omega}{\Omega_{e}}$

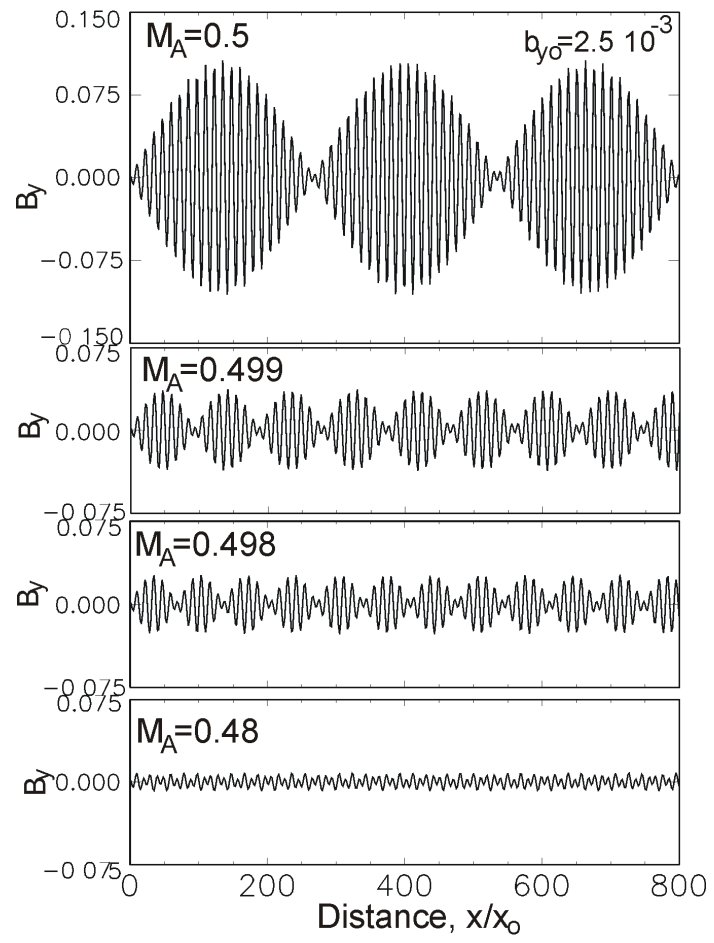

Fig. 13. Amplitude of periodic wave packets strongly decreases with deviation from $M_{A e}=1 / 2$.

$\left(\sqrt{\Omega_{p} \Omega_{e}}<\omega<\Omega_{e}\right)$ can be considered as Gendrin nonlinear modes which travel in a plasma like a soliton without significant distortions. Wave packets, consisting of a spectrum of the nonlinear Gendrin modes, are well guided by the magnetic field, and can propagate without significant reflection or trapping through a number of magnetic bottles associated with the mirror modes and give rise to the observed intermittent bursts of coherent whistlers in a broad region. Since the group speed of the wave packets is field-aligned, an azimuthal asymmetry of the waves relative to the symmetry axis of a mirror trough may be not uncommon. This can explain a difference in a wave activity recorded by different spacecraft crossing the same mirror structures.

\subsection{Modifications due to a finite electron temperature}

The results discussed in the previous section, however, cannot be applied directly to magnetosheath or magnetospheric plasmas. The conditions under which the nonlinear whistler wave packets can exist are modified in the presence of a finite electron temperature and temperature anisotropy which lead to the generation of whistler waves through the electron cyclotron instability. Unfortunately, analytical solution of nonlinear Vlasov equations is probably not feasible. Nevertheless some features of nonlinear whistler waves in a nonequilibrium Vlasov plasma can be predicted. The wavenumber (frequency) of the oscillations in the periodic whistler wave packets is approximately determined by the 


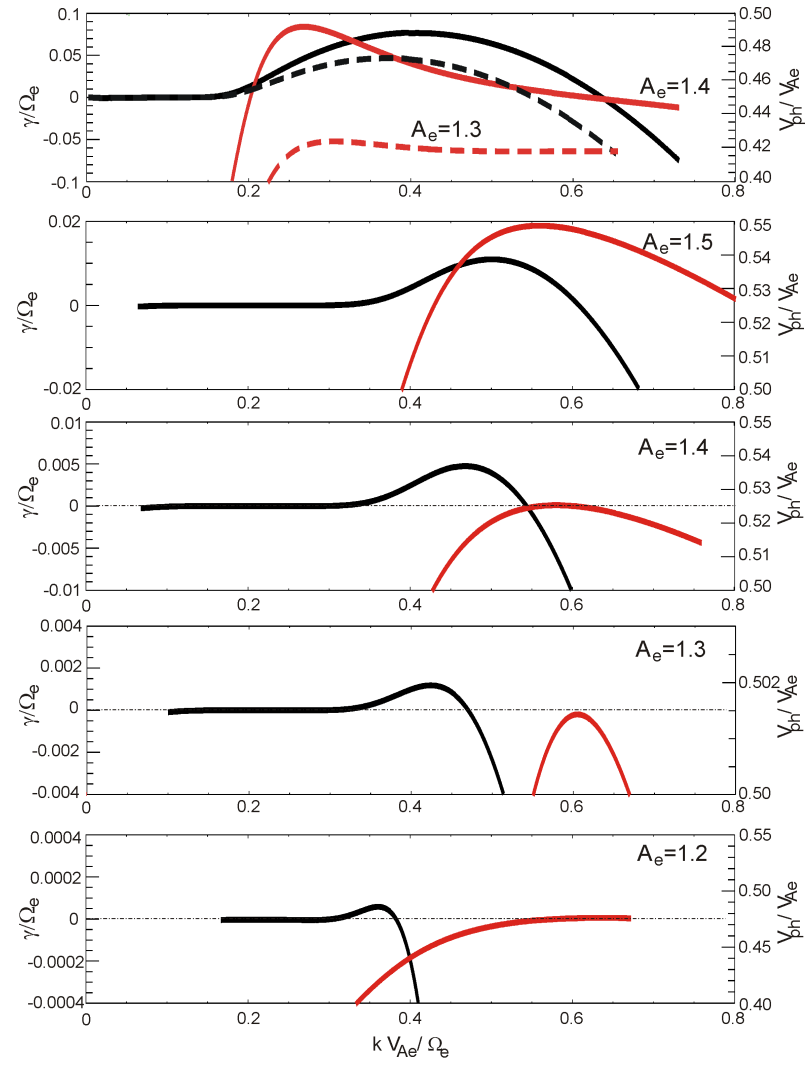

Fig. 14. Phase speed and the growth rate of whistlers generated by a temperature anisotropy instability.

wavenumber at the inflection point $(\omega / k=\partial \omega / \partial k)$ of the dispersion curve $(\omega, k)$ that corresponds to a maximum on the curve of the phase speed. The top panel of Fig. 14 shows the phase speeds (black curves) of whistlers propagating parallel to the magnetic field in a magnetosheath plasma with typical parameters for the flank crossings $\left(n=20 \mathrm{~cm}^{-3}\right.$, $B=9 \mathrm{nT}, T_{e \|}=T_{p \|}=100 \mathrm{eV}, T_{p \perp} / T_{p \|}=1.3, T_{e \perp} / T_{e \|}=1.4$ (solid curves) and 1.3 (the dashed curves). The red curves depict the growth rate of waves excited by the electron temperature anisotropy. The maximum of the phase speed shifts to $\omega \sim 0.2 \Omega_{e}$, which is in a reasonable agreement with the magnetosheath observations (Zhang et al., 1988; Baumjohann et al., 1999; Maksimovic et al., 2001). Note that, in general, this frequency does not coincide with the frequency at which the growth rate reaches the maximum value. Indeed, the comparison between the observed frequencies of the narrow-banded whistlers in the magnetosheath and the theoretical values corresponding to the maximum growth rate often shows a clear difference with a factor of $f_{\text {obs }} / f_{\text {theor }} \sim 2$ (Massood et al., 2006). Generation of Gendrin' nonlinear wave packets at different angles to the magnetic field could also lead to the different frequencies of the narrow-banded emissions. In a cold plasma, these frequencies must follow a simple relation $f_{\theta} / f_{0}=\cos \theta$, where $f_{\theta}\left(f_{0}\right)$ is the frequency of the whistler wave packets propagating at the angle $\theta\left(0^{\circ}\right)$, respectively. However, the observations do not show a such relationship although the frequency sometimes varies by a factor of 2 (see, e.g. Fig. 9a). Therefore, rather, variations in the observed frequency can arise as a result of different distant sources. Small scale variations in the total wave magnetic fields embedded within the solitary structures (see, e.g. Fig. 9b) are also difficult to explain in terms of the theory developed for the cold case.

\subsection{Electron dynamics}

The characteristic feature of the NR for whistler waves is the coherent gyrating motion of the particles participating in periodic momentum exchange. A shift of the pitch-angle of the trapped electrons from $90^{\circ}\left(270^{\circ}\right)$ to intermediate values observed during a wave activity might indicate that these electrons experience a bulk, coherent, gyrophase-bunched motion. The behavior of the electrons within mirror waves was studied by Chisham et al. (1998) using AMPTE observations. Chisham et al. (1998) observed a "cooling" of the electrons with pitch angles close to $90^{\circ}$ in the field troughs, and a "heating" of the electrons with pitch angles just greater than a trapping pitch-angle. A similar feature, i.e. a shift of the peak in the differential fluxes from $90^{\circ}$ to smaller angles is also observed in our study and cannot be explained by a pitch-angle diffusion of the electrons which are in the resonance with a whistler band.

Chisham et al. (1998) have interpreted this as a result of a different behavior of the "deeply" and "shallowly" trapped electrons undergoing a combination of betatron deceleration and Fermi acceleration (deceleration). In contrast, our model suggests that the electrons in the periodic nonlinear wave packets coherently move on spiral trajectories that leads to a shift of the maximum of differential fluxes from $90^{\circ}$ to intermediate pitch-angles. We can roughly estimate the pitch-angle of the gyrophase-bunched electrons assuming the equipartition of the momentum flux carried by different particle populations. The conservation of the momentum flux in the wave frame yields $m_{e} n_{t r} U u_{e \perp} \approx k \frac{b_{\perp} B_{x}}{\mu_{o}}$, where $k$ is a factor which takes into account the equipartition of total momentum flux of the particles, $n_{t r}$ is a number density of the trapped electrons, $U$ and $u_{e} \perp$ are, respectively, the wave speed and the transverse speed of the gyrating electrons, $b_{\perp}, B_{x}$, are the wave and ambient magnetic fields (Dubinin et al., 2003). Since $u_{e x} \sim U$ then the pitchangle $\alpha \approx \operatorname{atan}\left(\frac{u_{e \perp}}{u_{e x}}\right) \sim \frac{k}{\delta n M_{A e}^{2}} \frac{b_{\perp}}{B_{o}} \sim 30-45^{\circ}$. This value is in a reasonable agreement with the observations. Here $M_{A e}=U / V_{A e} \sim 0.5$ is the Mach number, $\delta n=\frac{n_{t r}}{n_{e}} \sim 0.03$ is a fraction of the trapped particles, $k \sim 1 / 3-1 / 4, \frac{b_{\perp}}{B_{o}} \sim 0.02$.

Note that a further thorough investigation is necessary to confirm the predicted signature of gyrophase-bunched 
resonant electrons. To date there are only a few observations in space plasmas of signatures of gyrophase-bunched electron distributions (e.g. Gurgiolo et al., 2000). It is worth noting that gyrophase-bunched ions are often observed upstream of the bow shock where the solar wind plasma contains an ion beam along the magnetic field. In this case, a similar nonlinear resonant system, consisting of the solar wind protons and beam ions, generates the coherent low-frequency waves and gyrophase-bunched motions of the ions (Dubinin et al., 2004).

\subsection{Wave amplitudes}

It is not clear yet what other components, besides the resonant electrons which form a small bulge on the distribution function, also participate in the nonlinear interaction mediated by whistler waves in a loss-cone plasma configuration. It could be, for example, the trapped resonant electrons characterized by the different temperatures $T_{\perp, r}, T_{\|, r}$, and the bulk electrons or it could be all particle populations, including the protons. The resonant properties of such a system may become weaker. This can be demonstrated by the example of a cold plasma with an electron beam parallel to the magnetic field. Recall that an electron beam-plasma system is linearly stable with respect to whistler waves if the temperature anisotropy is not added (see e.g. Hashimoto and Matsumoto, 1976). Nevertheless it is useful to analyze the nonlinear properties of a such system consisting of three interacting populations. Figure 15 shows how the structure of an "oscilliton" changes with an increase of the density of beam electrons. As the density of the beam increases the amplitude of wave oscillations decreases, i.e. the resonant characteristics of the system become weaker. Since the anisotropy and loss-cone instabilities are purely kinetic (not fluid) an assessment of the resonance properties of a such system and the contribution of the different particle populations can only be analyzed accurately by PIC simulations which are very desirable to study the generation of nonlinear coherent whistler wave structures.

Typically, observations in the magnetosheath and magnetosphere show approximately the same amplitudes of narrow-band whistler emissions despite there being significant difference in the number of resonant electrons which can generate these waves. Correspondingly, the growth rate of the electron temperature anisotropy instability strongly decreases for magnetospheric conditions. The panels $2-5$ from the top in Fig. 14 present the phase speed and the growth rate for whistlers under conditions typical for the magnetosphere observations, $n=0.6 \mathrm{~cm}^{-3}, B=9 \mathrm{nT}, T_{p \|}=3700 \mathrm{eV}, T_{e \|}=200 \mathrm{eV}$, $T_{p \perp} / T_{p \|}=1.4, T_{e \perp} / T_{e \|}=1.5(1.4,1.3,1.2)$. The growth rate becomes more than ten times less than its value for the magnetosheath parameters (the number of resonant electrons strongly decreases), and the maximum of the phase speed is observed at $\omega \sim k_{\max } U \sim 0.27,0.25,0.21,0.18 \Omega_{e}$ at

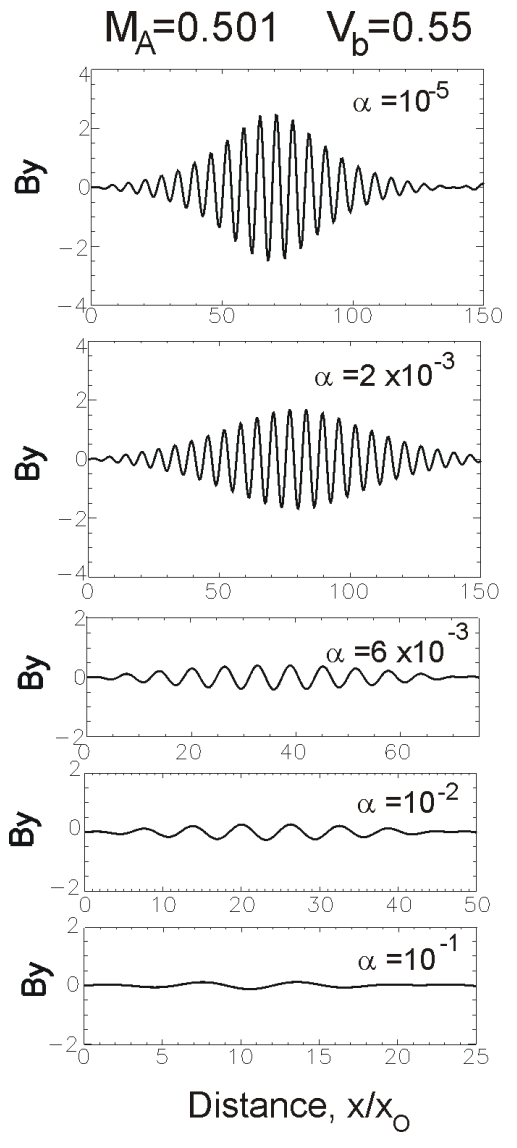

Fig. 15. Amplitude of oscillatory waves in a beam-plasma system at different values of the beam density $\alpha=n_{b} / n_{p}$ $\left(M_{A e}=0.501, V_{b}=0.55 V_{A e}\right.$.

$T_{e \perp} / T_{e \|}=1.5,1.4,1.3,1.2$, respectively. A damping also increases with anisotropy decreasing.

The similar intensity of narrow banded whistler emissions in two very different plasmas is difficult to explain in terms of linear anisotropy instabilities. We assume that the wave amplitude depends not only on the amount of free energy contained, for example, in the temperature anisotropy of the bulk or trapped populations, but also on the resonant properties of the system which can be different in plasmas with different $\beta_{e}$ and $T_{e \perp} / T_{e \|}$. The amount of free energy determines the behavior of the system at the linear stage. Due to pitch-angle scattering temperature anisotropy decreases and the system can evolve to a state of a marginal stability, similar as a system with ion temperature anisotropy unstable to the generation of ion cyclotron waves (Gary et al., 1997). Then nonlinear effects, with selection and amplification of "resonant" modes, may become dominant. This scenario must be tested and quantified in PIC simulations of plasma configurations with a temperature anisotropy and a loss cone. It is interesting to note that preliminary PIC simulations (Sydora and Sauer, private communication, 2006) confirm it. Sydora and Sauer (2006) studied whistler generation in a plasma with an 
electron temperature anisotropy. They found that at the initial state the system evolved in accordance with the predictions of linear theory. The amplitude of the waves centered at the frequency corresponding to the maximum growth rate $\gamma_{\max }$ exponentially increased with a characteristic time determined by $\gamma_{\max }^{-1}$ while the temperature anisotropy decreased. Then the system remains in a state with $T_{e \perp} / T_{e \|}$ equal to the value at which the marginal stability was reached, and wave packets of coherent emissions with the frequency close to the frequency of whistlers with the maximum phase speed are generated. With an increase of $\beta_{e}$ the amplitude of waves decreased and in the range of $\beta_{e} \approx 0.2-1$ the wave amplitudes became comparable.

\section{Conclusions}

In conclusion, the observations by Cluster of magnetospheric lion roars: sporadic narrow-band whistler emissions at $f \sim 0.1-0.2 f_{e}$, where $f_{e}$ is the electron gyrofrequency, found that their properties are very similar to the emissions observed in the magnetosheath. The waves are usually observed in the magnetic field troughs associated with mirror structures. The remarkable feature is that "whistler turbulence" consists of a train of periodic, almost coherent and monochromatic wave packets. The origin of coherency and wave packet structuring is discussed in terms of the nonlinear properties of whistlers. It is suggested that the observed wave packets are nonlinear, solitary like whistler structures. It is shown that Gendrin's wave modes, i.e. waves in a cold plasma at a frequency $f=\frac{f_{e}}{2} \cos \theta$ can be significantly nonlinearly amplified due to a periodic momentum exchange between the particles and fields, which generates the observed oscillations. Such a system has a nonlinear resonance at the frequency at which the phase speed equals the group speed. In a warm nonequilibrium plasma, due to the temperature anisotropy or loss-cone distribution, the resonant point on the dispersion curve shifts to $f \sim 0.1-0.2 f_{e}$. This is in a agreement with the observations. The model also predicts a gyrophase-bunched motion of the resonant electrons. The preliminary assessment of the electron dynamics confirms such electron behavior. However, some properties of the "kinetic" oscilliton-like structures, such as variations in frequency and oscillations in the total amplitude of the wave magnetic field, cannot be explained in the terms of a simple analogy with whistler oscillitons in a cold plasma.

The peak to peak amplitude of the magnetic and electric field variations in the waves is about $0.2 \mathrm{nT}$ and $0.5 \mathrm{mV} / \mathrm{m}$, respectively, which is also in close agreement with the typical amplitudes of similar structures in the magnetosheath. On the other hand, comparison of the bulk plasma parameters in both regions shows that a number of the resonant electrons which can drive these waves, is strongly different. In spite of the low number density of the magnetospheric plasma and, a correspondingly, low number density of resonant electrons, the amplitudes and the characteristic frequencies of magnetospheric lion roars appear similar to those in the sheath. This implies that the origin of the emissions and their characteristics are determined by nonlinear processes. It is assumed that the resonance properties of a complex nonlinear plasma system, with respect to the whistler generation, depend on $\beta_{e}$ and $T_{e \perp} / T_{e \|}$. To date it is not clear whether other parts of the distribution function, than that of the trapped electrons contribute to the interaction. In this respect, PIC simulations of whistler emission are very desirable for a closer comparison with the space observations.

The main features of narrow-band whistler emissions can be explained in terms of the nonlinear resonance of whistler waves: (a) the resonant frequency is determined by the value at which the group and phase speeds are equal; (b) the observed amplitude modulations, giving rise to the trains of wave packets is as effect of the nonlinear beating; (c) the amplitude of the waves is determined not only by an amount of a free energy but also by the resonant properties of the system (the number of the interacting populations and the number of particles which take part in the momentum exchange) (d) Gendrin's oblique whistler modes, for which the group speed is equal to the parallel component of the phase speed, can be taken from a pool of linearly unstable whistler waves and subsequently resonantly amplified; (e) nonlinear solitary Gendrin's whistler waves can readily propagate along the magnetic field to be observed far from the site of their generation; (f) a coherent oscillatory motion of particles which plays a part in the momentum exchange with other populations is an inherent feature of the nonlinear resonance.

Acknowledgements. E. M. Dubinin thanks the Deutsche Forschungsgemeinschaft and the Max-Planck-Gesellschaft for supporting this work by grant (WO 910/1-P). E. M. Dubinin wishes to express his acknowledgement to the Meudon Observatory (France) for their support during his visits.

Topical Editor I. A. Daglis thanks R. Thorne and another referee for their help in evaluating this paper.

\section{References}

Balogh, A., Dunlop, M. W., Cowley, S. W. H., Southwood, D. J., et al.: The Cluster magnetic field investigation, Space Sci. Rev., 76, 65-92, 1997.

Baumjohann, W., Treumann, R. A., Georgescu, E., et al.: Waveform and packet structure of lion roars, Ann. Geophys., 17, 15281534, 1999, http://www.ann-geophys.net/17/1528/1999/.

Baumjohann, W., Georgescu, E., Fornacon, K.-H., et al.: Magnetospheric lion roars, Ann. Geophys., 18, 406-410, 2000, http://www.ann-geophys.net/18/406/2000/.

Chisham, G. D., Burgess, D., Schwartz, S. J., and Dunlop, M. W.: Observations of electron distributions in magnetosheath mirror mode waves, J. Geophys. Res., 103, 26 765-26774, 1998. 
Cornilleau-Wehrlin, N., Chauveau, P., Louis, S., et al.: The Cluster Spatio-Temporal Analysis of Field Fluctuations (STAFF) experiment, Space Sci. Rev., 79, 107-136, 1997.

Cornilleau-Wehrlin, N., Chanteur, G., Perraut, S., Rezeau, L., et al.: First results obtained by the Cluster STAFF Experiment, Ann. Geophys., 21, 437-456, 2003, http://www.ann-geophys.net/21/437/2003/.

Dubinin E., Sauer, K., and McKenzie, J. F.: Nonlinear stationary whistler waves and whistler solitons (oscillitons). Exact solutions, J. Plasma. Phys., 69, 305-330, 2003.

Dubinin, E., Sauer, K., and McKenzie, J. F.: Nonlinear stationary waves and solitons in ion beam-plasma configurations, J. Geophys. Res., 109, A02208, doi:10.1029/2003JA010283, 2004.

Gary, S. P., Wang, J., Winske, D., and Fuselier, S. A.: Proton temperature anisotropy upper bound, J. Geophys. Res., 102, 27 159$27169,1997$.

Gendrin, R.: Le guidage des whistlers par le champ magnetique, Planet. Space Sci., 5(4), 274-282, 1961.

Gurgiolo, C., Larson, D., Lin, R. N., and Wong, H.: A gyrophasebunched electron signature upstream of the Earth's bow shock, Geophys. Res. Lett., 27, 19, 3153-3156, 2000.

Gustafsson, G., Bostrom, R., Holback, B., et al.: The Electric Field and Wave Experiment for the Mission, Space Sci. Rev., 79, 137156, 1997.

Hashimoto, K. and Matsumoto, H.: Temperature anisotropy and beam-type whistler instabilities, Phys. Fluids, 19, 1507-1512, 1976.

Johnstone, A. D., Alsop, C., Burge, S., et al.: PEACE: a Plasma Electron and Current Experiment, Space Sci. Rev., 79, 351, 1997.

Kennel, C. F. and Thorne, R. M.: Unstable growth of unducted whistlers propagating at an angle to the geomagnetic field, J. Geophys. Res., 72, 871-878, 1967.

Lee, L. C., Wu, C. S., and Price, C. P.: On the generation of magnetosheath lion roars, J. Geophys. Res, 92, 2343-2348, 1987.

Maksimovic, M., Harvey, C., Santolik, O., et al.: Polarization and propagation of lion roars in the dusk side magnetosheath, Ann. Geophys., 19, 1429-1438, 2001, http://www.ann-geophys.net/19/1429/2001/.

Masood, W., Schwartz, S., Maksimovic, M., and Fazakerley, A. N.: Electron velocity distribution and lion roars in the magnetosheath, Ann. Geophys., 24, 1725-1735, 2006, http://www.ann-geophys.net/24/1725/2006/.
McKenzie, J. F.: Radiation Losses from a test particle in a plasma, Phys. Fluids., 10(12), 2680-2694, 1967.

Rème, H., Bosqued, J. M., Sauvaud, J. A., Cros, A., et al.: The Cluster ion spectrometry (CIS) experiment, Space Sci. Rev., 79, 303-350, 1997.

Sauer K., Dubinin, E., and McKenzie, J. F.: Wave emission by whistler oscillitons: application to coherent lion roars, Geophys. Res. Lett., 29(24), 2226, doi:10.1029/2002GL015771, 2002.

Smith, E. J., Holzer, R. E., McLeod, M. G., and Russell, C. T.: Magnetic noise in the magnetosheath in the frequency range 3300 Hz, J. Geophys. Res., 72, 4803-4813, 1967.

Smith, E. J., Holzer, R. E., and Russell, C. T.: Magnetic emissions in the magnetosheath at frequencies near $100 \mathrm{~Hz}, \mathrm{~J}$. Geophys. Res, 74, 3027-3036, 1969.

Smith, E. J. and Tsurutani, B. T.: Magnetosheath lion roars, J. Geophys. Res, 81, 2261-2266, 1969.

Thorne, B. and Tsurutani, B. T.: The generation mechanism for magnetosheath lion roars, Nature, 293, 384-386, 1981.

Treumann, R. A., Georgescu, E., and Baumjohann, W.: Lion roar trapping in mirror modes, Geophys. Res. Lett, 27, 13, 18431846, 2000.

Tsurutani, B., Smith, E., Anderson, R., et al.: Lion roars and nonoscillatory drift mirror waves in the magnetosheath, J. Geophys. Res., 87, 6060-6072, 1982.

Webb, G. M., McKenzie, J. F., Dubinin, E., and Sauer, K.: Hamiltonian formulation of nonlinear travelling whistler waves, Nonlin. Processes Geophys., 12, 643-660, 2005, http://www.nonlin-processes-geophys.net/12/643/2005/.

Yearby, K. H., Alleyne, H., Cornilleau-Wehrlin, N., et al.: Observations of lion roars in the magnetosheath by the STAFF/DWP experiment on the Double Star TC1 spacecraft, Ann. Geophys., 23, 2861-2866, 2005, http://www.ann-geophys.net/23/2861/2005/.

Zhang, Y., Matsumoto, H., and Kojima, H.: Lion roars in the magnetosheath: The Geotail observations, J. Geophys. Res, 103, 4615-4626, 1998. 